\title{
Role of Indocyanine Green in Laparoscopic Cholecystectomy
}

\author{
George C Obonna ${ }^{1}$, Martin C Obonna², Rajneesh K Mishra ${ }^{3}$
}

\begin{abstract}
Background: The most feared complication during laparoscopic cholecystectomy (LC) is bile duct injury. Real-time intraoperative imaging using indocyanine green (ICG) reduces the risk of bile duct injury by improving visualization of the biliary tree during laparoscopy. This effect will also shorten operative time and hence reduce the dangers of prolonged operation time. It also subserves the diagnostic value in its use in the liver function test.

Aim: This study was aimed to elucidate the role of ICG as an investigative tool that aids the operative procedure of laparoscopic cholecystectomy. Materials and methods: The analysis of case series of ICG laparoscopic cholecystectomy in our hospital—the World Laparoscopic Hospital, Gurgaon, India.

Results: In all the cases, fluorescent cholangiography using intravenous injection of ICG has become the optimal tool to confirm the biliary tract anatomy during $\mathrm{LC}$ because it has potential advantages over radiographic cholangiography in that it does not require irradiation or dissection of the triangle of Calot. This early visualization of the cystic duct and additional imaging of the common bile duct (CBD) may increase safety in LC and offers an alternative to the intraoperative cholangiogram in patients with increased risk of CBD injury.

Conclusion: Laparoscopic cholecystectomy with real-time ICG fluorescence cholangiography enables a better visualization and identification of the biliary tree and therefore should be considered as a means of increasing the safety of LC.

Keywords: Acute cholecystitis, Indocyanine green cholangiography, Laparoscopic cholecystectomy.

World Journal of Laparoscopic Surgery (2020): 10.5005/jp-journals-10033-1388
\end{abstract}

\section{INTRODUCTION}

Laparoscopic cholecystectomy (LC) is the gold standard in the removal of the diseased gallbladder. Bile duct injury is rare with an incidence of $0.3-0.7 \%,{ }^{1}$ but it can lead to serious consequences. Surgery for gallbladder disease tends to be difficult for even experienced doctors and has a high risk of complications.

Intraoperative fluorescent imaging with indocyanine green (ICG) has been employed for confirming the potency of vascular reconstruction surgery, liver transplantation, ${ }^{2}$ anastomosis of the gastrointestinal tract (GIT), ${ }^{3}$ brain aneurysms,${ }^{4}$ identification of sentinel lymph mode navigation, ${ }^{5}$ and hepatocellular carcinoma detection. ${ }^{6}$ Recently, an intraoperative cholangiography technique in LC involving the excretion of fluorescent ICG in the bile after intravenous injection has been used to determine the bile duct anatomy. ${ }^{7-9}$

Currently, some detailed reports ${ }^{10,11}$ have been published on LC using intraoperative ICG cholangiography and suggested its safety and feasibility. In this study, we evaluated the process of intraoperative ICG cholangiography including LC for gallbladder disease.

Indocyanine green is a medical dye that subserves wide application especially in its use in biliary surgery. Its fluorescent properties under near-infrared light have been used in the intraoperative characterization of the biliary tree to ensure safe surgery during LC. It is a tricarbocyanine dye having a molecular weight of $751 \mathrm{Da}$.

\section{Materials and Methods}

Based on the procedures of ICG, LC was performed at the World Laparoscopy Hospital, India.

\section{Results}

Results show that there was positive and successful intraoperative identification of the extrahepatic bile ducts. This reduced the
${ }^{1}$ Department of Surgery, University of Medical Sciences Teaching Hospital, UNIMED Ondo, Nigeria

${ }^{2}$ Department of Anatomy, College of Medicine, Abia State University, Uturu, Nigeria

${ }^{3}$ Department of Minimal Access Surgery, World Laparoscopy Hospital, Gurugram, Haryana, India; Department Chair, Minimal Access Surgery, The Global Open University, Nagaland, India

Corresponding Author: George C Obonna, Department of Surgery, University of Medical Sciences Teaching Hospital, UNIMED Ondo, Nigeria, Phone: +234 8038584310, e-mail: obogeo2009@yahoo.com

How to cite this article: Obonna GC, Obonna MC, Mishra RK. Role of Indocyanine Green in Laparoscopic Cholecystectomy. World J Lap Surg 2020;13(1):1-3.

Source of support: Nil

Conflict of interest: None

likelihood of causing an injury to the bile ducts. This is, however, unlike the conventional imaging, whose results are usually not very successful. It has been noted that the intraoperative misidentification of the bile ducts anatomy is usually the main cause of bile duct injury.

\section{Discussion}

Indocyanine green is also known as the florescent dye. This dye has been used since 1956. Aurogreen trade mark is injected intravenously 45 minutes before surgery. It is actually the one that helps light the path for better and real-time identification of the biliary anatomy during LC. There is a florescent imaging system that is usually used together with a laparoscope. The system must have a lightning system that provides light for both infrared and xenon rays. Figure 1 shows the package of ICG. Figure 2 demonstrated 


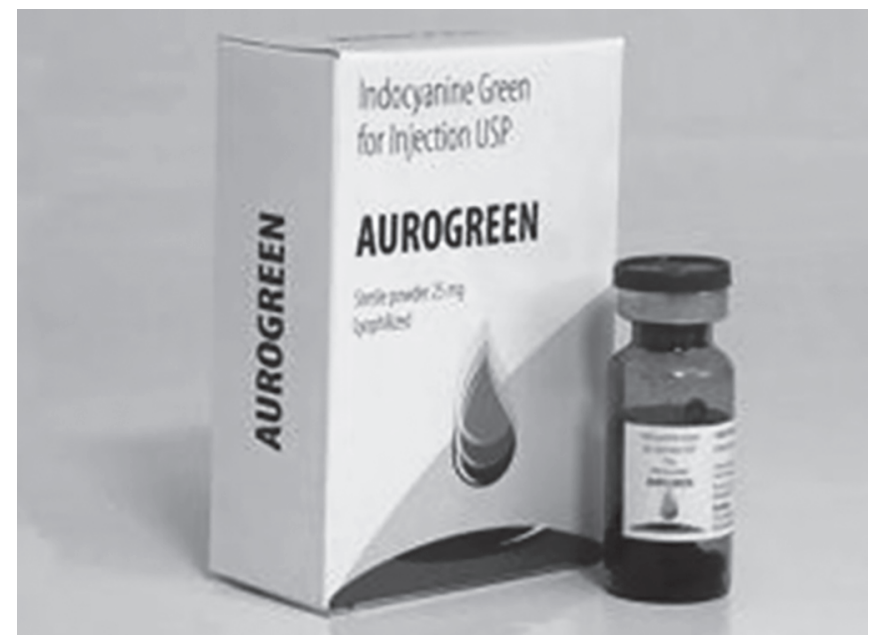

Fig. 1: Showing a preparation of indocyanine green

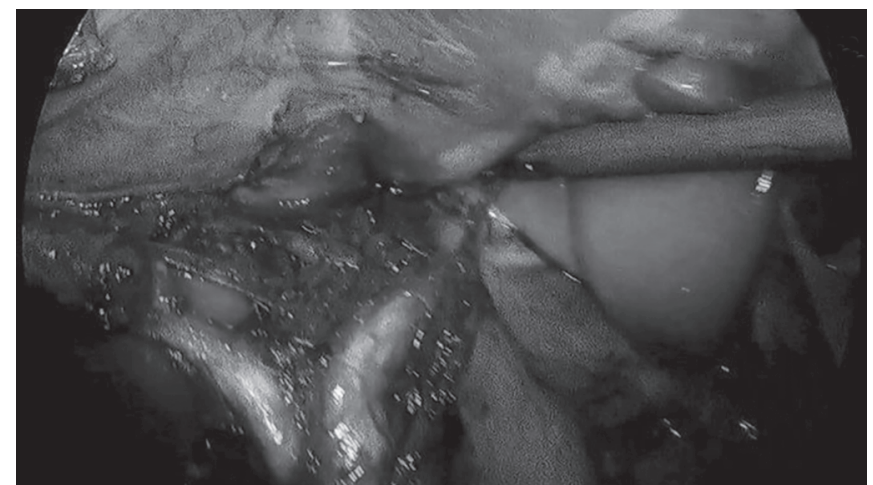

Fig 3: Fluorescent mapping showing cystic, right and left hepatic duct

the highlight near infrared cholangiography and also as shown in Figure 3 is the road map of enhanced fluorescent imaging at LC.

Indocyanine green acts as an imaging agent, which is sterile, water soluble, and has a peak spectral absorption averaging at 805 $\mathrm{nm}$ in blood pressure or simply the blood.

It does not undergo enterohepatic recirculation. It only stays in the bile for about 8 minutes after injection. The removal of ICG depends on several factors, including the blood flow of the liver, biliary excretion, and parenchymal cellular function. It is contraindicated in those having iodine toxicity. Sometimes, florescence may not be detected 45 minutes after the injection of ICG. Therefore, when this happens, the second dose of $2.5 \mu \mathrm{g}$ of ICG can be administered. Also a second injection of ICG can be given if there is anything regarding perfusion that cannot be clearly understood during the surgery. Apart from its role to analyze the extrahepatic biliary anatomy better, thus reducing the incidence of bile duct injury, it also plays a role in identifying the anatomic structure in a quick manner, thereby reducing the time used in performing the procedure of cholangiography and hence also shortening the entire duration of surgery.

There is also no need to bring onboard additional equipment and manpower, especially the radiological personnel for an X-ray.

The technique of ICG also plays a role as a teaching tool for practitioners who are able to identify the relevant extrahepatic

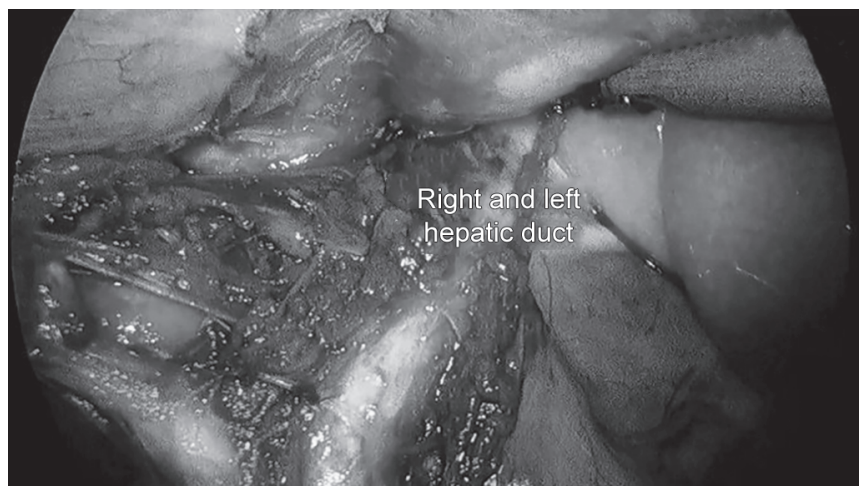

Fig. 2: Fluorescent mapping showing common bile duct and cystic duct

structures in almost every patient. This makes them experienced with the procedure that makes it easier for them to perform future procedure.

It does not require X-ray, thus no one is exposed to radiation. The ICG that is used is usually safe and does not cause any adverse reaction in patients. It is also cost-effective.

\section{Conclusion}

Real-time near-infrared fluorescence cholangiography offers better technology that can help with the identification of the vital biliary anatomy.

It helps to reduce the risk of bile duct injury by providing clear vision of the vital extrahepatic structure. The reduced risk helps to avoid complications that can arise from the injury of the bile duct, which sometimes can be fatal to a patient.

\section{References}

1. Ishizawa T, Bandai Y, ljichi M, et al. Fluorescent cholangiography illuminating the biliary tree during laparoscopic cholecystectomy. Dr J Surg 2010;97(9):1369-1377. DOI: 10.1002/bjs.7125.

2. Kubota K, Kita J, Shimoda M, et al. Intraoperative assessment of reconstructed vessels in living-donor liver transplantation, using a novel fluorescence imaging technique. J Hepatobilliary Pancreat Surg 2006;13(2):100-104. DOI: 10.1007/s00534-005-1014-z.

3. Saito T, Yano M, Motoori M, et al. Subtotal gastrectomy for gastric tube cancer after esophagectomy; safe procedure preserving the proximal part of gastric tube based on intraoperative ICG blood flow evaluation. J Surgoncol 2012;106(1):107-110. DOI: 10.1002/jso. 23050.

4. Raabe A, Beck J, Gerlach R, et al. Near infrared indocyanine green video angiography; a new method for intraoperative assessment of vascular flow. Neurosurgery 2003;52(1):132-139. DOI: 10.1227/00006123-200301000-00017.

5. Ohdaira H, Nimura $\mathrm{H}$, Mitsumori $\mathrm{N}$, et al. Validity of modified gastrectomy combined with sentinel node navigation surgery for early gastric cancer. Gastric Cancer 2007;10(2):117-122. DOI: 10.1007/ s10120-007-0419-6.

6. Gotoh K, Yamada T, Ishikawa O, et al. A novel image guided surgery of hepatocellular carcinoma by indocyanine green fluorescence imaging navigation. J Surgoncol 2009;100(1):75-79. DOI: 10.1002/ jso.21272.

7. Mitsuhashi N, Kimura F, Shimizu H, et al. Usefulness of intraoperative fluorescence imaging to evaluate local anatomy in hepatobiliary surgery. J Hepatobiliary Pancreat Surg 2008;15(5):508-514. DOI: 10.1007/s00534-007-1307-5. 
8. Tagaya N, Shimoda M, Kato M, et al. Intraoperative exploration of biliary anatomy using fluorescence imaging of indocyanine green in experimental and clinical cholecystectomies. J Hepatobiliary Pancreat Sci 2010;17(5):595-600. DOI: 10.1007/s00534-009-0195-2.

9. Aoki T, Murakami M, Yasuda D, et al. Intraoperative fluorescent imaging using indocyanine green for liver mapping and cholangiography. J Hepatobiliary PancreatSci 2010;17(5):590-594. DOI: 10.1007/s00534009-0197-0.
10. Schools RM, Connell NJ. Stassen near infrared fluorescence imaging for real time intraoperative anatomical guidance in minimally invasive surgery: a systematic review of the literature. World J Surg 2015;39(5):1069-1079. DOI: 10.1007/s00268-014-2911-6.

11. Vlek SL, Van Dam DA, Rubinstein SM, et al. Biliary tract visualization using near infrared imaging with indocyanine green during laparoscopic cholecystectomy: results of a systematic review. Surg Endosc 2017(7):2731-2742. DOI: 10.1007/s00464-016-5318-7. 